\title{
Quasi-copulas and signed measures
}

\section{Roger B. Nelsen}

Department of Mathematical Sciences, Lewis \& Clark College, Portland (USA)

\section{José Juan Quesada-Molina}

Department of Applied Mathematics, University of Granada (Spain)

\section{José Antonio Rodríguez-Lallena \\ Manuel Úbeda-Flores}

Department of Statistics and Applied Mathematics, University of Almería (Spain)

\section{Main goals:}

- Study of (bivariate) quasi-copulas with fractal mass distributions.

- Study of the mass distribution of $W^{n}$ - the point-wise best-possible lower bound for the set of $n$-quasi-copulas (and $n$-copulas).

- As a consequence, not every multivariate quasi-copula induces a signed measure on $[0,1]^{n}$. 


\section{Copulas and Quasi-copulas}

- The importance of copulas: Sklar's Theorem [6,8]. The joint distribution function $H$ of a random vector $\left(X_{1}, X_{2}, \cdots, X_{n}\right)$ with respective univariate margins $F_{1}, F_{2}, \cdots, F_{n}$, can be expressed in terms of an $n$-copula $C$ that is uniquely determined on $\times_{i=1}^{n}$ Range $F_{i}$ in the form

$$
H(\mathbf{x})=C\left(F_{1}\left(x_{1}\right), F_{2}\left(x_{2}\right), \cdots, F_{n}\left(x_{n}\right)\right), \quad \mathbf{x} \in[-\infty, \infty]^{n} .
$$

- The notion of quasi-copula was introduced [1] in order to characterize operations on distribution functions that can, or cannot, be derived from operations on random variables defined on the same probability space.

Theorem 1 [2] An n-dimensional quasi-copula (or n-quasi-copula) is a function $Q:[0,1]^{n} \rightarrow[0,1]$ that satisfies:

(Q1) $Q\left(u_{1}, \cdots, u_{i-1}, 0, u_{i+1}, \cdots, u_{n}\right)=0$ and $Q\left(1, \cdots, 1, u_{i}, 1, \cdots, 1\right)=u_{i}$ for all $\mathbf{u} \in[0,1]^{n}$ and for every $i \in\{1,2, \cdots, n\}$;

(Q2) $Q$ is nondecreasing in each variable; and

(Q3) the Lipschitz condition: $|Q(\mathbf{u})-Q(\mathbf{v})| \leq \sum_{i=1}^{n}\left|u_{i}-v_{i}\right| \forall \mathbf{u}, \mathbf{v} \in[0,1]^{n}$. 
- Every $n$-copula is an $n$-quasi-copula; and when $Q$ is an $n$-quasi-copula but not an $n$-copula, it is said that $Q$ is a proper $n$-quasi-copula.

- Every $n$-quasi-copula $Q$ satisfies that, for every $\mathbf{u} \in[0,1]^{n}$,

$$
W^{n}(\mathbf{u})=\max \left(0, \sum_{i=1}^{n} u_{i}-n+1\right) \leq Q(\mathbf{u}) \leq \min (\mathbf{u})=M^{n}(\mathbf{u}) .
$$

- $M^{n}$ is an $n$-copula for all $n \geq 2, W^{2}$ is a 2-copula, and $W^{n}(n \geq 3)$ is a proper $n$-quasi-copula.

- For an $n$-quasi-copula $Q$ and an $n$-box $B=\times_{i=1}^{n}\left[a_{i}, b_{i}\right]$ in $[0,1]^{n}$, the $Q$-volume of $B$ is defined similarly than for $n$-copulas, i.e.,

$$
V_{Q}(B)=\sum \operatorname{sgn}(\mathbf{c}) \cdot Q(\mathbf{c})
$$

We refer to $V_{Q}$ as the mass distribution of $Q$, and $V_{Q}(B)$ the mass accumulated by $Q$ on $B$.

The importance of quasi-copulas:

- Quasi-copulas (and copulas) are a special type of aggregation operators.

- The set of quasi-copulas is a complete lattice. 


\section{Signed measures}

- Every $n$-copula $C$ induces a positive measure $\mu_{C}$ defined on the Lebesgue $\sigma$ algebra for $[0,1]^{n}$.

- Let $\lambda_{n}$ denote the Lebesgue measure in $\mathbb{R}^{n}$. The measure $\mu_{C}$ is stochastic, i.e., for every Lebesgue measurable (L-m) set $A$ in $[0,1]$

$$
\mu_{C}\left([0,1]^{i-1} \times A \times[0,1]^{n-i}\right)=\lambda_{1}(A) .
$$

- $\mu_{C}$ is characterized by the fact that $\mu_{C}(B)=V_{C}(B)$ for every $n$-box $B$.

Definition 1 [5] A signed measure $\mu$ on a measurable space $(S, \mathcal{A})$ is an extended real valued, countably additive set function on the $\sigma$-algebra $\mathcal{A}$ such that $\mu(\emptyset)=$ 0 , and such that $\mu$ assumes at most one of the values $+\infty$ and $-\infty$.

- Many proper $n$-quasi-copulas $Q$ induce signed measures $\mu_{Q}$ on $[0,1]^{n}$ in the sense that $\mu_{Q}(B)=V_{Q}(B)$ for every $n$-box $B$. Such measures must satisfy:

(a) for every L-m set $A$ in $[0,1], \mu_{Q}\left([0,1]^{i-1} \times A \times[0,1]^{n-i}\right)=\lambda_{1}(A)$,

(b) for every $\mathbf{u} \in[0,1]^{n}$, if $u_{i} \leq v_{i} \leq 1$ for some $i=1,2, \cdots, n$, then

$$
0 \leq \mu_{Q}\left(\left[0, u_{1}\right] \times \cdots \times\left[0, u_{i-1}\right] \times\left[u_{i}, v_{i}\right] \times\left[0, u_{i+1}\right] \times \cdots \times\left[0, u_{n}\right]\right) \leq v_{i}-u_{i}
$$


Example 1 Let $s_{1}, s_{2}$ and $s_{3}$ be three segments in $[0,1]^{2}$, respectively defined by $f_{1}(x)=x+1 / 3$ if $x \in[0,2 / 3], f_{2}(x)=x$ if $x \in[1 / 3,2 / 3]$, and $f_{3}(x)=$ $x-1 / 3$ if $x \in[1 / 3,1]$. We spread a mass of $2 / 3$ uniformly on each of $s_{1}$ and $s_{3}$, and a mass of $-1 / 3$ uniformly on $s_{2}$. Let $(u, v) \in[0,1]^{2}$. If we define $Q(u, v)$ as the net mass in the 2-box $[0, u] \times[0, v]$, then $Q$ is a 2-quasi-copula. To be exact: $Q(u, v)=\min (u, v, \max (0, u+v-1, u-1 / 3, v-1 / 3)) \quad \forall(u, v) \in[0,1]^{2}$.

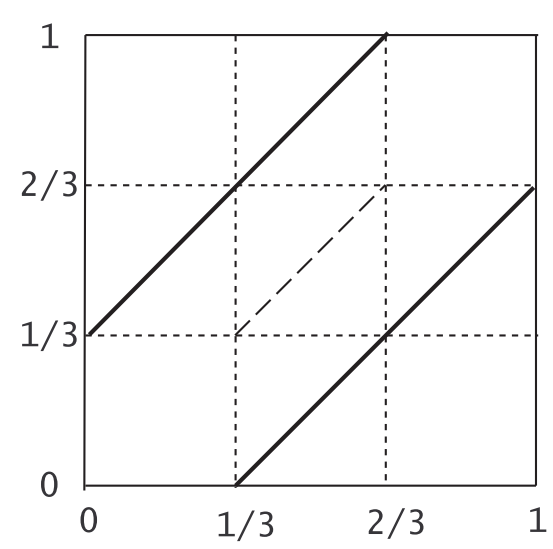

Thus, there exists a signed measure $\mu_{Q}$ such that $\mu_{Q}(B)=V_{Q}(B)$ for every 2-box $B$ in $[0,1]^{2}$ (the difference between the positive measure $\mu_{Q}^{+}$obtained by spreading a mass of $2 / 3$ uniformly on each of $s_{1}$ and $s_{3}$, and the positive measure $\mu_{Q}^{-}$obtained by spreading a mass of $1 / 3$ uniformly on $\left.s_{2}\right)$. 


\section{Bivariate quasi-copulas with fractal mass dis- tributions}

In [4], the authors construct families of 2-copulas whose supports are fractals [3] by using an iterated function system. We now extend some of the results in [4] to the case of quasi-copulas.

Definition 2 A quasi-transformation matrix is a matrix $T=\left(t_{i j}\right), i=$ $1,2, \cdots, m$ and $j=1,2, \cdots, n$, with the column index first and the rows ordered from bottom to top, with entries between $-1 / 3$ and 1 , for which the sum of the entries is 1, no row or column has every entry zero, the negative entries are not in the first and the last row or column, e.g. $t_{1 j}, t_{m j}, t_{i 1}$, and $t_{i n}$ for all $i=1,2, \cdots, m, j=1,2, \cdots, n$, and the sum of the entries in any submatrix of the form $\left(t_{i j}\right)$ for $i=a, a+1, \cdots, b, j=c, c+1, \cdots, d$ is nonnegative when $a=1$ or $b=1$ or $c=m$ or $d=n$.

Example 2 Let $T$ be the quasi-transformation matrix given by

$$
T=\left(\begin{array}{ccc}
0 & 1 / 3 & 0 \\
1 / 3 & -1 / 3 & 1 / 3 \\
0 & 1 / 3 & 0
\end{array}\right)
$$


Let $R_{i j}=\left[p_{i-1}, p_{i}\right] \times\left[q_{j-1}, q_{j}\right]$ be a 2-box in $[0,1]^{2}$ such that $p_{i}$ (respectively, $q_{j}$ ) denotes the sum of the entries in the first $i$ columns (respectively, $j$ rows) of $T$. Then, for any 2-quasi-copula $Q$, let $T(Q)$ be the 2-quasi-copula which, for each $(i, j)$, spreads mass $t_{i j}$ on $R_{i j}$ in the same (but re-scaled) way in which $Q$ spreads mass on $[0,1]^{2}$, i.e.,

$$
\begin{aligned}
T(Q)(u, v)= & \sum_{i^{\prime}<i, j^{\prime}<j} t_{i^{\prime} j^{\prime}}+\frac{u-p_{i-1}}{p_{i}-p_{i-1}} \cdot \sum_{j^{\prime}<j} t_{i j^{\prime}}+\frac{v-q_{j-1}}{q_{j}-q_{j-1}} \cdot \sum_{i^{\prime}<i} t_{i^{\prime} j} \\
& +t_{i j} \cdot Q\left(\frac{u-p_{i-1}}{p_{i}-p_{i-1}}, \frac{v-q_{j-1}}{q_{j}-q_{j-1}}\right),
\end{aligned}
$$

where empty sums are defined to be zero.

Definition 3 Let $T$ be a quasi-transformation matrix. For any 2-quasi-copula $Q$, we define $T^{m}(Q)=T\left(T^{m-1}(Q)\right), m=1,2, \cdots$, where $T^{0}(Q)=Q$.

Theorem 2 For each quasi-transformation matrix $T \neq(1)$, there is a unique 2-quasi-copula $Q_{T}$ for which $T\left(Q_{T}\right)=Q_{T}$. Moreover, $Q_{T}$ is the limit of the sequence $\left\{Q, T(Q), T^{2}(Q), \cdots\right\}$ for any 2-quasi-copula $Q$. 
Let $T$ be the quasi-transformation matrix given by (1), i.e.,

$$
T=\left(\begin{array}{ccc}
0 & 1 / 3 & 0 \\
1 / 3 & -1 / 3 & 1 / 3 \\
0 & 1 / 3 & 0
\end{array}\right)
$$

and let $\Pi^{2}$ be the copula of independent random variables, i.e., $\Pi^{2}(u, v)=u v$ for all $(u, v) \in[0,1]^{2}$. The mass distributions of $T\left(\Pi^{2}\right)$ and $T^{2}\left(\Pi^{2}\right)$ :
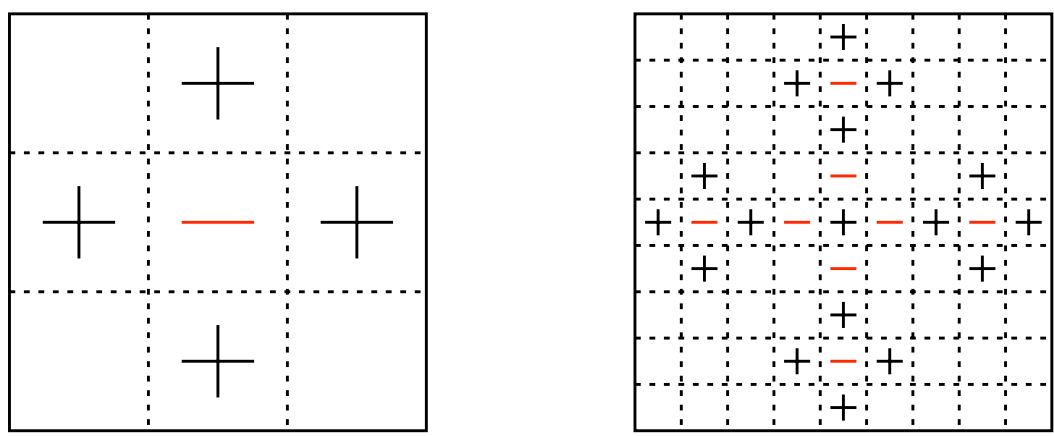
For each iteration $m \geq 1$ :

- $R_{m}^{+}=3^{m}+\sum_{i=0}^{m-1} 3^{i} \cdot 5^{m-i-1}$ (number of 2-boxes with (+) mass).

- $R_{m}^{-}=5^{m}-3^{m}-\sum_{i=0}^{m-1} 3^{i} \cdot 5^{m-i-1}$ (number of 2-boxes with (-) mass).

- Total (+) mass $T^{m}\left(\Pi^{2}\right): 1+(1 / 5) \sum_{i=0}^{m-1}(5 / 3)^{m-i}$.

- Total (-) mass $T^{m}\left(\Pi^{2}\right):-(1 / 5) \sum_{i=0}^{m-1}(5 / 3)^{m-i}$.

- $V_{T^{m}\left(\Pi^{2}\right)}\left([1 / 3,2 / 3]^{2}\right)=-1 / 3 \Rightarrow T^{m}\left(\Pi^{2}\right)$ is a proper 2-quasi-copula.

Let $\Pi_{T}^{2}=\lim _{m \rightarrow \infty} T^{m}\left(\Pi^{2}\right)$.

- $V_{\Pi_{T}^{2}}\left([1 / 3,2 / 3]^{2}\right)=-1 / 3 \Rightarrow \Pi_{T}^{2}$ is a proper 2-quasi-copula.

- Total (+) mass of $\Pi_{T}^{2}:+\infty$.

- Total (-) mass of $\Pi_{T}^{2}:-\infty$.

- $\lim _{m \rightarrow \infty} \lambda_{2}\left(R_{m}^{+}\right)=\lim _{m \rightarrow \infty} \lambda_{2}\left(R_{m}^{-}\right)=0$. 


\section{The mass distribution of $W^{n}$}

For any integer $k \geq 2$, let $T_{k}=\{1,2, \cdots, k\}$. We divide $[0,1]^{n}$ in the $k^{n}$ following $n$-boxes:

$$
R\left(i_{1}, i_{2}, \cdots, i_{n}\right)=\prod_{j=1}^{n}\left[\frac{i_{j}-1}{k}, \frac{i_{j}}{k}\right],\left(i_{1}, i_{2}, \cdots, i_{n}\right) \in T_{k}^{n} .
$$

Theorem 3 Let $n$ and $k$ be two integers such that $k \geq n-1 \geq 1$. Let $m=$ $\sum_{j=1}^{n} i_{j}-k(n-1)$. Then

$$
V_{W^{n}}\left(R\left(i_{1}, i_{2}, \cdots, i_{n}\right)\right)= \begin{cases}\frac{(-1)^{m-1}}{k} \cdot\left(\begin{array}{c}
n-2 \\
m-1
\end{array}\right), & 1 \leq m \leq n-1, \\
0, & \text { otherwise. }\end{cases}
$$

Moreover, for each $m$, with $1 \leq m \leq n-1$, the number of $n$-boxes $R\left(i_{1}, i_{2}, \cdots, i_{n}\right)$ satisfying that $V_{W^{n}}\left(R\left(i_{1}, i_{2}, \cdots, i_{n}\right)\right)=\frac{(-1)^{m-1}}{k}\left(\begin{array}{c}n-2 \\ m-1\end{array}\right)$ is $\left(\begin{array}{c}n-1+k-m \\ n-1\end{array}\right)$. 
Theorem 4 Let $n$ be an integer such that $n \geq 3$, and let $M$ be any positive real number. Then, there exists a finite set of n-boxes $\left\{J_{1}, J_{2}, \cdots, J_{p}\right\}$ in $[0,1]^{n}$ whose interiors are pairwise disjoint and such that
(a) $\sum_{i=1}^{p} V_{W^{n}}\left(J_{i}\right)>M$, and
(b) $\sum_{i=1}^{p} \lambda_{n}\left(J_{i}\right)<1 / M$;

similarly, we can also find a finite set of n-boxes $\left\{J_{1}^{\prime}, J_{2}^{\prime}, \cdots, J_{q}^{\prime}\right\}$ in $[0,1]^{n}$ with pairwise disjoint interiors such that
(c) $\sum_{i=1}^{q} V_{W^{n}}\left(J_{i}^{\prime}\right)<-M$, and
(d) $\sum_{i=1}^{q} \lambda_{n}\left(J_{i}^{\prime}\right)<1 / M$.

Corollary 5 For every $n \geq 3, W^{n}$ does not induce a signed measure on $[0,1]^{n}$. 


\section{References}

[1] Alsina, C., Nelsen, R.B., Schweizer, B. (1993) On the characterization of a class of binary operations on distribution functions. Statist. Probab. Lett. 17, $85-89$.

[2] Cuculescu, I., Theodorescu, R. (2001) Copulas: diagonals and tracks. Rev. Roumaine Math. Pures Appl. 46, 731-742.

[3] Edgar, G.A. (1990) Measure, Topology, and Fractal Geometry. Springer, New York.

[4] Fredricks, G.A., Nelsen, R.B., Rodríguez-Lallena, J.A. (2005) Copulas with fractal supports. Insurance Math. Economics 37, 42-48.

[5] Halmos, P.R. (1974) Measure Theory. Springer, New York.

[6] Nelsen, R.B. (2006) An Introduction to Copulas. Second Edition. Springer, New York.

[7] Sklar, A. (1959) Fonctions de répartition à $n$ dimensions et leurs marges. Publ. Inst. Statist. Univ. Paris 8, 229-231. 